\title{
Article \\ Conversion Therapy of Intrahepatic Cholangiocarcinoma Is Associated with Improved Prognosis and Verified by a Case of Patient-Derived Organoid
}

\author{
Zhiwei Wang ${ }^{1,+}$, Yun Jin ${ }^{1,+}$, Yinghao Guo ${ }^{1}$, Zhenhua Tan ${ }^{1}$, Xiaoxiao Zhang ${ }^{1}{ }^{\circledR}$, Dan Ye ${ }^{1}$, Yuanquan Yu ${ }^{1}$, \\ Shuyou Peng ${ }^{1}$, Lei Zheng ${ }^{2, *}$ and Jiangtao $\mathrm{Li}^{1}{ }^{1, *}$
}

1 Department of Surgery, Second Affiliated Hospital, Zhejiang University School of Medicine, Hangzhou 310009, China; wangzw824@zju.edu.cn (Z.W.); 2515045@zju.edu.cn (Y.J.); guoyinghao07@zju.edu.cn (Y.G.); tanzh99@163.com (Z.T.); zxiaoxiao204@163.com (X.Z.); 2316030@zju.edu.cn (D.Y.); zjuyyq@zju.edu.cn (Y.Y.); zrwkpsy@zju.edu.cn (S.P.)

2 Department of Oncology, Sidney Kimmel Cancer Center at Johns Hopkins University School of Medicine, Baltimore, MD 21231, USA

* $\quad$ Correspondence: lzheng6@jhmi.edu (L.Z.); zrlj@@zju.edu.cn (J.L.); Tel.: +86-571-89713727 (J.L.)

+ These authors contributed equally to this work.

Citation: Wang, Z.; Jin, Y.; Guo, Y.; Tan, Z.; Zhang, X.; Ye, D.; Yu, Y.; Peng, S.; Zheng, L.; Li, J. Conversion Therapy of Intrahepatic Cholangiocarcinoma Is Associated with Improved Prognosis and Verified by a Case of Patient-Derived Organoid. Cancers 2021, 13, 1179. https://doi.org/10.3390/ cancers13051179

Academic Editors: Ghassan Abou-Alfa and Masahito Shimizu

Received: 14 January 2021

Accepted: 8 March 2021

Published: 9 March 2021

Publisher's Note: MDPI stays neutral with regard to jurisdictional claims in published maps and institutional affiliations.

Copyright: (c) 2021 by the authors. Licensee MDPI, Basel, Switzerland. This article is an open access article distributed under the terms and conditions of the Creative Commons Attribution (CC BY) license (https:// creativecommons.org/licenses/by/ $4.0 /)$.
Simple Summary: The first core tip of this study is that we summarized the experience of conversion therapy of intrahepatic cholangiocarcinoma (IHCC) in a single center and found conversion therapy could provide a significant improvement in the overall survival of patients with IHCC. Previous studies mainly focused on case reports and case series, and our research provided more evidence for the efficacy of conversion therapy in IHCC. The second is that we established the organoid of IHCC for drug screening and firstly verified the results of drug screening tests in cancer organoid were consistent with the levels of efficacy observed in the patient from whom it was derived. Cancer organoid is a promising technology for conversion therapy according to our study but more organoids are still needed.

Abstract: This study was performed to determine the efficacy of conversion therapy in intrahepatic cholangiocarcinoma (IHCC) and explore the feasibility of cancer organoid to direct the conversion therapy of IHCC. Patient data were retrospectively reviewed in this study and cancer organoids were established using tissues obtained from two patients. A total of 42 patients with IHCC received conversion therapy, 9 of whom were downstaged successfully, and another 157 patients were initially resectable. Kaplan-Meier curves showed that the successfully downstaged patients had a significantly improved overall survival compared to those in whom downstaging was unsuccessful $(p=0.017)$, and had a similar overall survival to that of initially resectable patients $(p=0.965)$. The IHCC organoid was successfully established from one of two obtained tissues. Routine hematoxylin and eosin staining and immunohistological staining found the organoid retained the histopathological characteristics of the original tissues. Whole exome sequencing results indicated the IHCC organoid retained appropriately $87 \%$ of the variants in the original tissue. Gemcitabine and paclitaxel exhibited the strongest inhibitory effects on the cancer organoid as determined using drug screening tests, consistent with the levels of efficacy observed in the patient from whom it was derived. This study indicates that conversion therapy could improve the survival of patients with IHCC despite its low success rate, and it may be directed by cancer organoids though this is merely a proof of feasibility.

Keywords: intrahepatic cholangiocarcinoma; conversion therapy; organoid; overall survival; genetic profiles; drug screening

\section{Introduction}

Intrahepatic cholangiocarcinoma (IHCC) is a rare and highly aggressive epithelial cancer accounting for $10-15 \%$ of all primary malignant liver tumors [1]. The incidence 
of IHCC has increased during the past two decades and shows a rise in the rate of mortality [2]. To date, surgical resection is the only potentially effective treatment for IHCC. However, the recurrence rates after resection have been reported to range from $40-80 \%$ [3]. Moreover, most patients with IHCC are diagnosed at an advanced stage owing to the lack of specific symptoms, rendering them unsuitable for surgical resection [4]. In patients with unresectable IHCC, conventional chemotherapy and radiotherapy are possible treatment options; however, these techniques are met with limited efficacy. Generally, the five-year survival of patients with IHCC is less than $5 \%[5,6]$.

To improve overall survival, several novel approaches have been proposed in recent decades. The concept of conversion therapy was proposed by Bismuth H. et al. in 1996, which demonstrated that initially unresectable malignant tumors can be downstaged using systemic or local treatment, such that patients could likely undergo surgical resection [7]. This technique was first used in the treatment of colorectal cancers (CRC) and is currently used widely in the treatment of various cancers including gastric and pancreatic cancers (PC) and hepatocellular carcinoma (HCC) [8-10]. Recently, the efficacy of conversion therapy in patients with IHCC was verified using several studies, which indicated that adequate tumor downstaging was achieved by systemic or local treatment and patients could obtain a relatively satisfactory prognosis [11-17]. However, most of these studies are case reports or case series containing no more than 10 patients. Thus, the overall success rate of conversion therapy is still unclear. Edeline J. et al. reported that only $22 \%$ of patients with locally advanced IHCC receiving radioembolization plus chemotherapy could be successfully downstaged to surgical resection owing to the low response rate of IHCC [18]. Therefore, the efficacy of conversion therapy in IHCC needs to be further improved.

Appropriate in vitro models of cancer have been explored for over 100 years. Conventional two-dimensional (2D) culture systems force cancer cells to adhere to an artificial substrate, which restricts the formation of a multi-dimensional structure. The formation of a monolayer morphology and the lack of contact with other normal cells makes it nearly impossible to engineer cancer cells to mimic the important characteristics of cancer tissues in vivo [19]. A novel three-dimensional (3D) culture system, also called an organoid, has been developed in recent times to address the defects of a 2D culture system. Compared to the 2D culture system, organoid technology provides a suitable extracellular matrix as a scaffold for the attachment of cells to form a multi-dimensional structure [20]. Consequently, cancer organoids can mimic the structure and function of original cancer tissues, including CRC, PC, and HCC [21-23]. High-throughput or medium-throughput drug screening of original cancer tissues is possible using organoids [24]. However, organoids of IHCC have been established, evaluated, and reported only in a few studies [23]. There remain uncertainties regarding whether a cancer organoid can be successfully used to determine and direct the anti-tumor treatment of IHCC, especially conversion therapy.

This study was undertaken to determine the efficacy of conversion therapy in patients with IHCC based on a retrospective review. Moreover, a cancer organoid was established to determine the feasibility of directing the conversion therapy of IHCC.

\section{Materials and Methods}

\subsection{Patient Selection}

Patients who were diagnosed with IHCC between January 2013 and June 2020, in the Second Affiliated Hospital, Zhejiang University, School of Medicine, were retrospectively reviewed. The following patients were excluded from this study: (1) those with remote lymph node metastases, large vessel invasion, or diffuse multiple lesions, (2) those not receiving any specific anti-tumor treatment, (3) those suffering from several types of cancers, and (4) those who had received anti-tumor treatment at other medical centers. This study was performed in compliance with the principles of the Declaration of Helsinki and was approved by the ethics committee of the Second Affiliated Hospital, Zhejiang University, School of Medicine (No. 2019-408). Informed consent from the included patients was not obtained owing to the retrospective nature of this study. 


\subsection{Data Collection}

To analyze the relationship between treatment patterns and prognosis in patients with IHCC, the demographics (age and gender), details on liver cirrhosis, number of lesions, tumor size, vascular invasion, perineural invasion, histological grade, AJCC TNM stage, residual tumor after surgical resection, perioperative adjuvant treatment, systemic treatment, and imaging examination date of the included patients were collected at admission. Patients were divided into initially resectable group, successfully downstaged group, and unsuccessfully downstaged group. All data were collected by two independent investigators and conflicts, if any, were resolved through consultation.

\subsection{Follow-Up}

Considering the relatively short survival time of patients with IHCC, all included patients were followed up for at least three months. The recurrence and metastasis of IHCC were recorded during follow up.

\subsection{Organoid Establishment}

Two tissue samples of resected IHCC were collected from two independent patients whose tumors were downstaged successfully after conversion therapy. The tissues were used to establish patient-derived cancer organoids to determine their effectiveness in directing the treatment pattern in conversion therapy. The experimental procedures were approved by the ethics committee of the Second Affiliated Hospital, Zhejiang University, School of Medicine, and informed consent from patients was obtained before tissue collection.

The obtained tissues were shipped on ice within $4 \mathrm{~h}$. The tumor tissues were cut into small pieces (appropriately 1-2 $\mathrm{mm}^{3}$ ) and incubated in $2 \mathrm{mg} / \mathrm{mL}$ of collagenase $\mathrm{D}$ (Roche, Basel, Switzerland) for $2 \mathrm{~h}$ at $37^{\circ} \mathrm{C}$. The isolated cells were passed through a $100-\mu \mathrm{M}$ cell strainer (Corning, Basel, Switzerland), pelleted using centrifugation at $300 \times g$ for $5 \mathrm{~min}$ at room temperature, and embedded in a mixture of Matrigel matrix (growth factor reduced; Corning, Corning, NY, USA) and culture medium on ice in a 1:1 ratio.

Appropriately $2-5 \times 10^{5}$ cells in $100 \mu \mathrm{L}$ of Matrigel matrix mixture were seeded into 24-well plates (Corning, Corning, NY, USA) after the plates were coated using $200 \mu \mathrm{L}$ of the Matrigel matrix mixture. Then, cells were overlaid with $500 \mu \mathrm{L}$ of culture medium. The culture medium contained advanced Dulbecco's modified Eagle medium/F12 (Gibco, Carlsbad, CA, USA) supplemented with $1 \times$ penicillin/streptomycin (ThermoFisher, Waltham, MA, USA), $1 \times$ Glutamax (ThermoFisher, Waltham, MA, USA), $10 \mathrm{mM}$ N-2-Hydroxyethylpiperazine-N-2-Ethane Sulfonic Acid (ThermoFisher, Waltham, MA, USA), $1 \times$ B27 supplement (Gibco, Carlsbad, CA, USA), $1 \times$ N2 supplement (Gibco, Carlsbad, CA, USA), $10 \mathrm{mM}$ nicotinamide (Sigma, St. Louis, MO, USA), $1.25 \mathrm{mM}$ N-acetylL-cysteine (Sigma, St. Louis, MO, USA), $10 \mathrm{nM}$ gastrin (Sigma, St. Louis, MO, USA), 5 $\mu \mathrm{M}$ A83-01 (Tocris, Bristol, UK), $50 \mathrm{ng} / \mathrm{mL}$ recombinant human epidermal growth factor (PeproTech, Rocky Hill, NJ, USA), $100 \mathrm{ng} / \mathrm{mL}$ recombinant human fibroblast growth factor 10 (PeproTech, Rocky Hill, NJ, USA), $10 \mu$ M Y-27632 (Tocris, Bristol, UK), 500 ng/mL recombinant human R-Spondin1 (PeproTech, Rocky Hill, NJ, USA), 10\% Afamin/Wnt3a CM (MBL Life Science, Kyoto, Japan), and $100 \mathrm{ng} / \mathrm{mL}$ recombinant human Noggin (PeproTech, Rocky Hill, NJ, USA). The culture medium was replaced every 4-5 days.

\subsection{Preparation of Histological Sections}

Primary tissue samples were fixed in $10 \%$ neutral-buffered formalin and embedded in paraffin blocks using a standard procedure. Organoids were released from the Matrigel Matrix by incubating them in the Cell Recovery Solution (Corning, Corning, NY, USA) for $1 \mathrm{~h}$, according to the manufacturer's instructions. Organoids were centrifuged at $300 \times g$ for $5 \mathrm{~min}$ at room temperature and fixed in 10\% neutral-buffered formalin for $2 \mathrm{~h}$. Next, the organoids were embedded into $4 \%$ low-melting agarose after centrifugation, and the agarose blocks were embedded into paraffin. Sections of primary tissues and organoids (4- $\mu \mathrm{M}$ thick) were prepared and subjected to routine hematoxylin and eosin (H\&E) staining 
and immunohistological staining. For immunohistological staining, mouse monoclonal antibodies against cytokeratin-7 (CK7, Abcam, Cambridge, UK, 1:2000) and epithelial cell adhesion molecule (EpCAM, Abcam, Cambridge, UK, 1:500) were used.

\subsection{Whole Exome Sequencing (WES)}

Genomic DNA of primary tissues and organoids were extracted using GenElute Mammalian Genomic DNA miniprep kits (Sigma, St. Louis, MO, USA). The quality of the extracted genomic DNA was verified by monitoring DNA degradation using $1 \%$ agarose gels and measuring the DNA concentration using Qubit DNA Assay kit (Invitrogen, Carlsbad, CA, USA). The exome sequences were enriched from $0.4 \mu \mathrm{g}$ of genomic DNA using an Agilent liquid capture system (Agilent SureSelect Human All Exon V6) according to the manufacturer's guidelines. Clustering was performed using a cBot Cluster Generation System and an Illumina PE Cluster kit (Illumina, San Diego, CA, USA) according to the manufacturer's guidelines. Sequencing was performed using an Illumina platform and $150 \mathrm{bp}$ paired-end reads were generated.

\subsection{Drug Treatment}

The cancer organoids were released from the Matrigel matrix by incubating in the Cell Recovery Solution (Corning, Corning, NY, USA) for $1 \mathrm{~h}$ followed by the incubation in $0.25 \%$ trypsin-ethylene diamine tetraacetic acid solution to allow its dissociation into single cells. These single cells were resuspended in Matrigel Matrix mixture on ice and approximately 500-1000 cells were embedded in a 384-well plate. The cells were left undisturbed to recover for 2 days. Then, the culture medium was replaced with fresh medium containing 0.01, 0.1, 1,10 , or $50 \mu \mathrm{M}$ of gemcitabine, 5-fluorouracil (5-FU), cisplatin, paclitaxel, infigratinib, or ivosidenib. After incubation with different drugs for $72 \mathrm{~h}$, the viability of cancer cells was determined using a CellTiter-Glo 3D Cell-Viability assay (Promega, Madison, WI, USA). The viability of cancer cells incubated in a culture medium with phosphate-buffered saline served as a negative control. The screening of each drug was performed in triplicate.

\subsection{Statistical Analysis}

Continuous variables in this study are expressed as medians with interquartile ranges and compared using the Mann-Whitney $U$ test. Categorical variables are expressed as a number using percentages and compared using the Chi-squared test. The Kaplan-Meier method and log-rank test were used to compare differences in overall survival among successfully downstaged, unsuccessfully downstaged, and initially resectable patients. SPSS Statistics 22.0 (IBM Corporation, New York, NY, USA) was used for statistical analysis and a two-tailed $p$-value of $<0.05$ was considered statistically significant.

\section{Results}

\subsection{Patient Characteristics}

Patients with IHCC admitted to our hospital between January 2013 and June 2020 were retrospectively reviewed as depicted in the flow chart (Figure 1). A total of 357 patients were diagnosed with IHCC, of which 108 were excluded from this study based on the exclusion criteria. There were 162 initially resectable and 87 initially unresectable patients among the remaining 249 patients. Forty-five initially unresectable patients had distant metastasis at the time of diagnosis, 27 and 18 of whom received palliative adjuvant therapy and palliative surgical resection, respectively. The remaining 42 of the 87 patients received conversion therapy, of which $9(21.4 \%)$ were downstaged successfully to further receive curative surgical resection. 


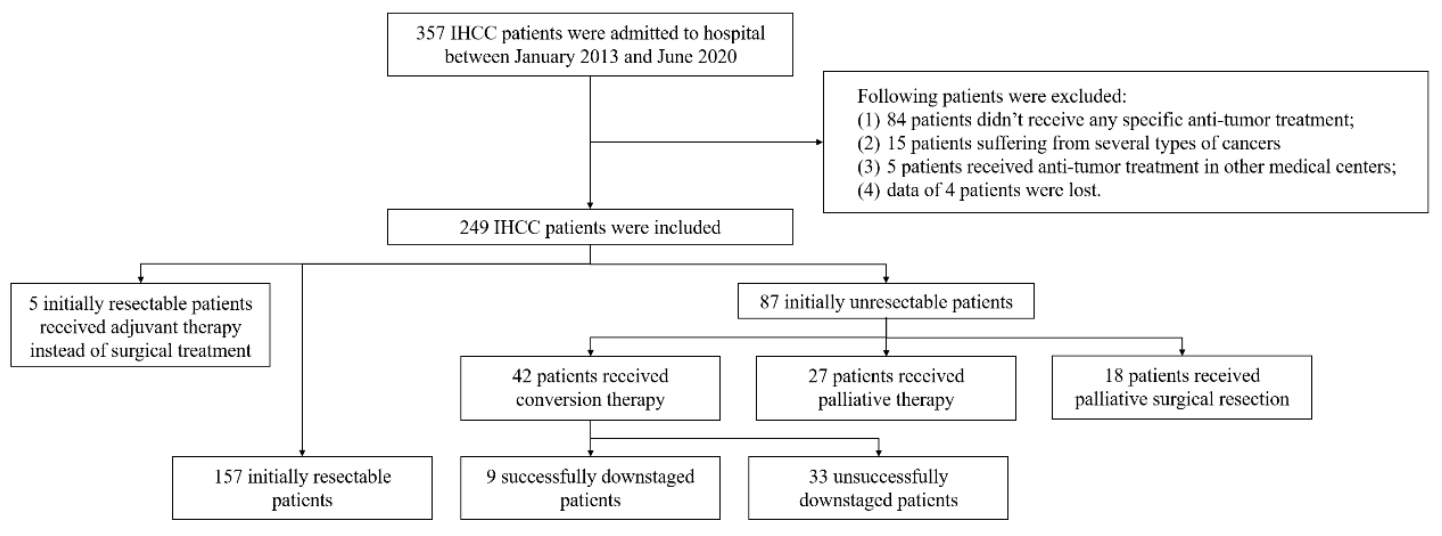

Figure 1. Flow chart of this study.

The data of patients receiving conversion therapy are listed in Table 1. No significant differences were found with respect to age, gender, liver cirrhosis, multiple lesions, vascular invasion, and perineural invasion between successfully downstaged and unsuccessfully downstaged patients. Most tumors of enrolled patients were poorly differentiated; however, histological grades of 20 unsuccessfully downstaged patients $(60.6 \%)$ were unknown owing to insufficient diagnosis after obtaining biopsy samples using fine-needle aspiration. Moreover, there were several T2-stage tumors in the unsuccessfully downstaged group (23 patients, 69.7\%) than in the successfully downstaged group (2 patients, $22.2 \%$ ).

Table 1. Demographic and clinical data of patients receiving conversion therapy.

\begin{tabular}{|c|c|c|c|}
\hline Variables & $\begin{array}{l}\text { Successfully Downstaged } \\
\text { Patients }\end{array}$ & $\begin{array}{c}\text { Unsuccessfully } \\
\text { Downstaged Patients }\end{array}$ & $p$ Value \\
\hline Number & 9 & 33 & \\
\hline Age, years & $64(61-71)$ & $62(52.5-68.5)$ & 0.450 \\
\hline Gender & & & 0.784 \\
\hline Male & $5(55.6 \%)$ & $20(60.6 \%)$ & \\
\hline Female & $4(44.4 \%)$ & $13(39.4 \%)$ & \\
\hline Liver cirrhosis & $1(11.1 \%)$ & $5(15.2 \%)$ & 0.759 \\
\hline Multiple lesions $(\geq 2)$ & $2(22.2 \%)$ & $20(60.6 \%)$ & 0.062 \\
\hline Tumor size & & & 0.655 \\
\hline$<5 \mathrm{~cm}$ & $1(11.1 \%)$ & $8(24.2 \%)$ & \\
\hline$\geq 5 \mathrm{~cm}$ & $8(88.9 \%)$ & $25(75.8 \%)$ & \\
\hline Vascular invasion & $3(33.3 \%)$ & $20(60.6 \%)$ & 0.257 \\
\hline Perineural invasion & $1(11.1 \%)$ & $0(0 \%)$ & 0.214 \\
\hline Histological grade & & & 0.004 \\
\hline Well & $0(0 \%)$ & $1(3.0 \%)$ & \\
\hline Moderate & $1(11.1 \%)$ & $0(0 \%)$ & \\
\hline Poor & $8(88.9 \%)$ & $12(36.4 \%)$ & \\
\hline Unknown & $0(0 \%)$ & $20(60.6 \%)$ & \\
\hline AJCC T stage & & & 0.008 \\
\hline T1a & $1(11.1 \%)$ & $2(6.1 \%)$ & \\
\hline $\mathrm{T} 1 \mathrm{~b}$ & $3(33.3 \%)$ & $0(0 \%)$ & \\
\hline $\mathrm{T} 2$ & $2(22.2 \%)$ & $23(69.7 \%)$ & \\
\hline T3 & $1(11.1 \%)$ & $5(15.2 \%)$ & \\
\hline $\mathrm{T} 4$ & $2(22.2 \%)$ & $5(15.2 \%)$ & \\
\hline AJCC N stage & & & 0.593 \\
\hline No & $3(33.3 \%)$ & $4(12.1 \%)$ & \\
\hline $\mathrm{N} 1$ & $6(66.7 \%)$ & $29(87.9 \%)$ & \\
\hline AJCC M stage & & & 1.000 \\
\hline M0 & $9(100 \%)$ & $33(100 \%)$ & \\
\hline M1 & $0(0 \%)$ & $0(0 \%)$ & \\
\hline
\end{tabular}

AJCC: American Joint Committee on Cancer. 


\subsection{Survival Analysis}

The detailed characteristics of patients who were downstaged successfully are presented in Table 2. Transcatheter arterial chemoembolization was used as the treatment protocol of conversion therapy in two patients. One of the patients received radiotherapy for the tumor surrounding the large vessels. The remaining six patients received gemcitabine-based chemotherapy to downstage the tumor, whereas one patient received toripalimab in addition to gemcitabine-based chemotherapy.

Table 2. Detailed characteristics of successfully downstaged patients by conversion therapy.

\begin{tabular}{|c|c|c|c|c|c|c|c|c|c|c|}
\hline Patient No. & Age & Gender & $\begin{array}{l}\text { Tumor } \\
\text { Size }(\mathrm{cm})\end{array}$ & $\begin{array}{l}\text { Histological } \\
\text { Grade }\end{array}$ & Tnm Stage & Treatment Pattern & $\begin{array}{l}\text { Radial } \\
\text { Margin } \\
\text { Status }\end{array}$ & $\begin{array}{c}\text { Recurrence } \\
\text { Time } \\
\text { (Months) }\end{array}$ & Status & $\begin{array}{c}\text { Survival } \\
\text { Time } \\
\text { (Months) }\end{array}$ \\
\hline Patient 1 & 73 & Female & 5.8 & Poor & T1bN0M0 & TACE & R0 & No & Alive & 84 \\
\hline Patient 2 & 44 & Male & 3 & Poor & T1aN1M0 & Gemcitabine & R0 & No & Alive & 8 \\
\hline Patient 3 & 72 & Male & 8.9 & Poor & T3N1M0 & Gemcitabine+Oxaliplatin & R0 & 29 & Dead & 39 \\
\hline Patient 4 & 62 & Female & 6 & Moderate & T4NOM0 & Radiotherapy & R0 & 21 & Alive & 21 \\
\hline Patient 5 & 64 & Female & 6.6 & Poor & T1bN1M0 & TACE & R0 & No & Alive & 30 \\
\hline Patient 6 & 70 & Female & 5 & Poor & T1bN1M0 & $\begin{array}{c}\text { Gemcitabine+Oxaliplatin+ } \\
\text { Toripalimab }\end{array}$ & R0 & No & Alive & 8 \\
\hline Patient 7 & 67 & Male & 5.5 & Poor & T2N0M0 & Gemcitabine+Cisplatin & R0 & 6 & Alive & 11 \\
\hline Patient 8 & 62 & Male & 8.6 & Poor & T2N1M0 & $\begin{array}{l}\text { Gemcitabine+albumin-bound } \\
\text { paclitaxel }\end{array}$ & R0 & No & Alive & 5 \\
\hline Patient 9 & 60 & Male & 9.2 & Poor & T4N1M0 & $\begin{array}{c}\text { Gemcitabine+albumin-bound } \\
\text { paclitaxel }\end{array}$ & R0 & No & Dead & 3 \\
\hline
\end{tabular}

TACE: transcatheter arterial chemoembolization.

On the other hand, detailed conversion strategies in 33 unsuccessfully downstaged patients were as follows: 13 patients receiving gemcitabine-based chemotherapy, 5 patients receiving transcatheter arterial chemoembolization, 4 patients receiving radiofrequency ablation, 4 patients receiving 5 -fluorouracil monotherapy, 2 patients receiving radiotherapy, 2 patients receiving immunotherapy, 1 patient receiving targeted therapy, and 2 patients receiving immunotherapy combined with targeted therapy.

All patients were followed up for at least 3 months. Two patients succumbed during the follow-up period, one of whom succumbed 39 months after diagnosis owing to lung metastasis of cancer, while the other succumbed to postoperative complications. An overall survival curve was generated based on the Kaplan-Meier method and log-rank test as shown in Figure 2. There was a significant improvement in the overall survival of successfully downstaged patients compared to the unsuccessfully downstaged patients $(p=0.017)$. Moreover, successfully downstaged patients had an overall survival similar to that of initially resectable patients $(p=0.965)$.
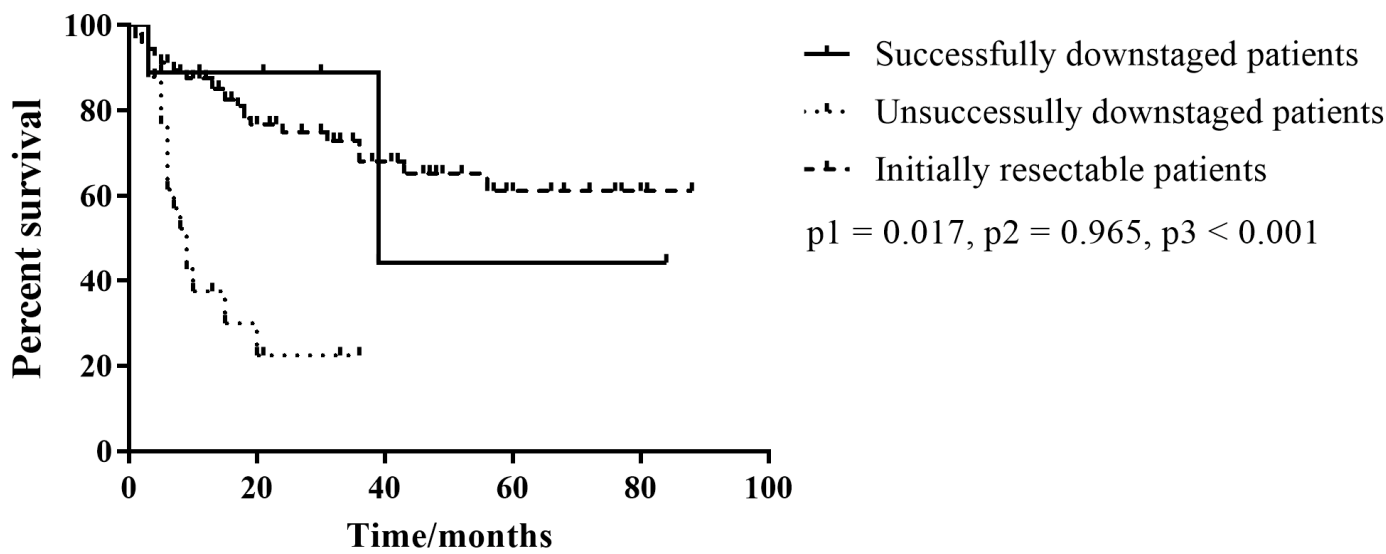

$\mathrm{p} 1=0.017, \mathrm{p} 2=0.965, \mathrm{p} 3<0.001$

Figure 2. Kaplan-Meier curves comparing overall survival of successfully downstaged patients, unsuccessfully downstaged patients, and initially resectable patients based on the log-rank test. p1: successfully downstaged patients versus unsuccessfully downstaged patients; p2: successfully downstaged patients versus initially resectable patients; p3: unsuccessfully downstaged patients versus initially resectable patients. 


\subsection{Organoid Establishment}

To determine the effectiveness of cancer organoids in developing a treatment pattern in IHCC conversion therapy, a cancer tissue was collected from patient 8 and patient 9 to establish cancer organoids. However, one of the cancer organoids from patient 9 was not established successfully owing to the extent of necrosis in the collected sample. Gross specimen and H\&E staining results of the other IHCC tissue are shown in Figure 3A,B. There were remnant nests of malignant cells in the obtained IHCC tissue owing to effective conversion therapy. Cancer organoid of this IHCC tissue was successfully established and was found to exhibit a monolayered cystic structure as seen in the bright field image (Figure 3C). H\&E staining of the cancer organoid (Figure 3D) suggested that it could mimic the structure and function of the original IHCC tissues. Some specific tumoral markers were tested by immunohistological staining, such as CK7 and EpCAM, as shown in Figure 4. It was found that expression profiles of cancer organoid resembled original tissue. CK7 and EpCAM were highly expressed in both organoid and tissue.

WES was performed by extracting the total DNA of the cancer organoid and original tissue to determine if the organoid retained the gene expression profiles of the original tissue. The circos plots of the original tissues and cancer organoids depicted in Figure 5A,B show a similar distribution. The distribution of base substitutions in the organoid and original tissue is shown in Figure 5C; both indicate overexpression of $\mathrm{T}>\mathrm{C} / \mathrm{A}>\mathrm{G}$ and $\mathrm{C}>\mathrm{T} / \mathrm{G}>\mathrm{A}$ transversions, followed by $C>G / G>C$ and $C>A / G>T$. In terms of the global variant profile, the IHCC organoid was found to retain appropriately $87 \%$ of the variants as that of the original tissue. In detail, $89 \%$ of the single nucleotide variants (shown in Figure 5D) and $82 \%$ of indels (not shown) in the original tissue were well retained in the IHCC organoid. Additionally, some representative genetic alterations can be seen in Figure 5E. Both organoid and original tissue harbored the MET, ARID1A, MUC5B, SMAD7, and IHD1 missense variants, an MSH3 non-frameshift insertion, and a MAP3K1 non-frameshift deletion.

A

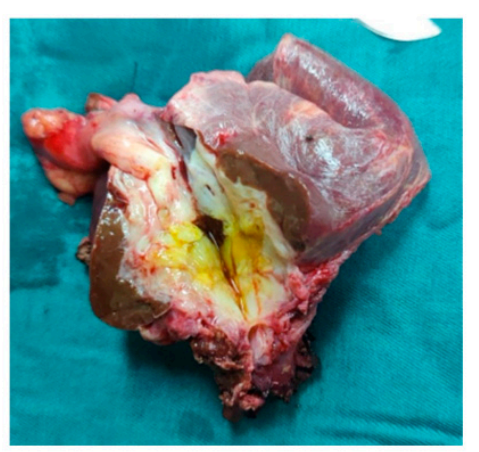

$\mathrm{C}$

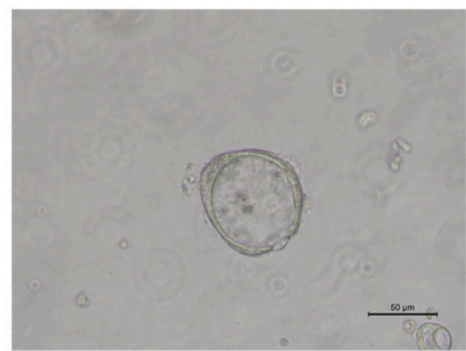

$\mathrm{B}$

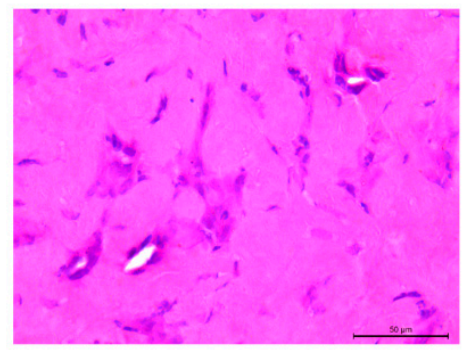

$\mathrm{D}$

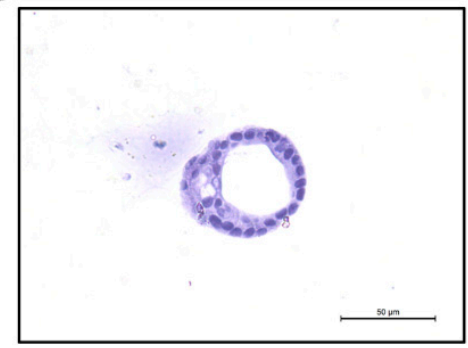

Figure 3. Histopathological characterization of cancer organoid. (A) Gross specimen and (B) H\&E staining of intrahepatic cholangiocarcinoma (IHCC) tissue, and (C) bright-field image and (D) H\&E staining of the cancer organoid. Scale bar, $50 \mu \mathrm{M}$. 


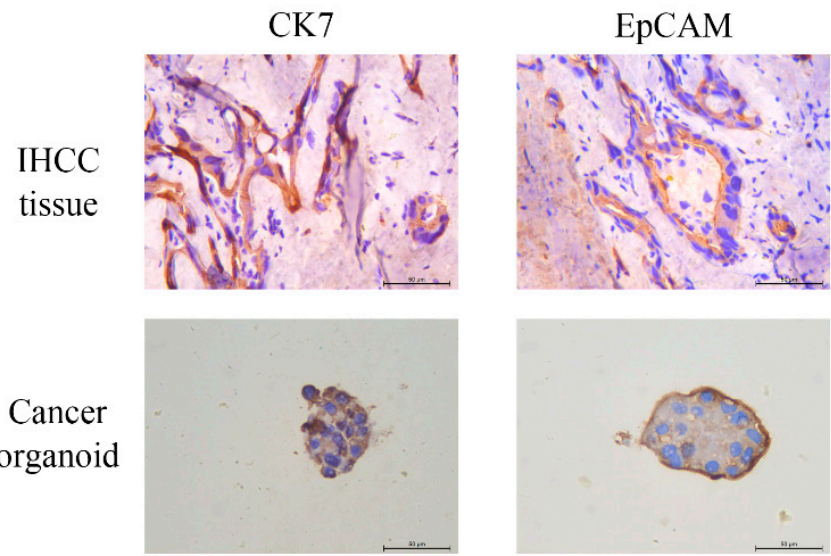

Figure 4. Immunohistological staining of IHCC tissue and cancer organoid, including CK7 and EpCAM. Scale bar, $50 \mu \mathrm{M}$.

A

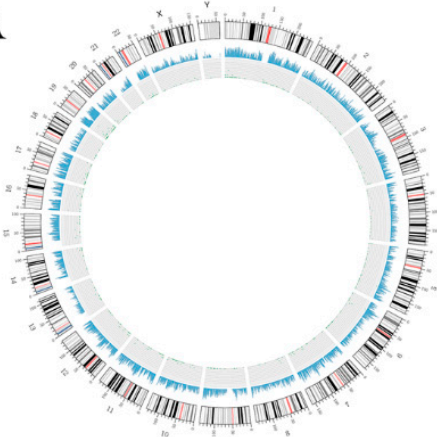

Original tissue

C

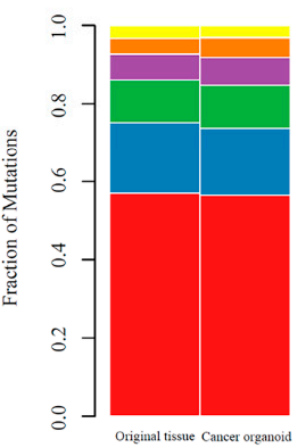

B

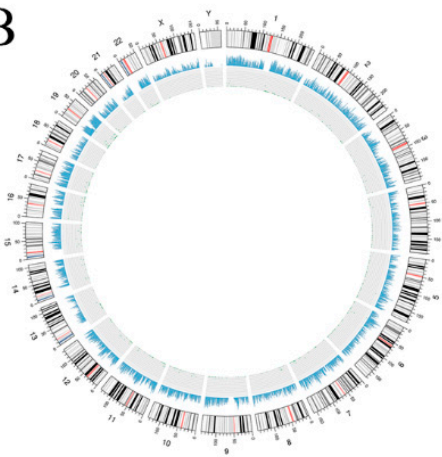

Cancer organoid

D

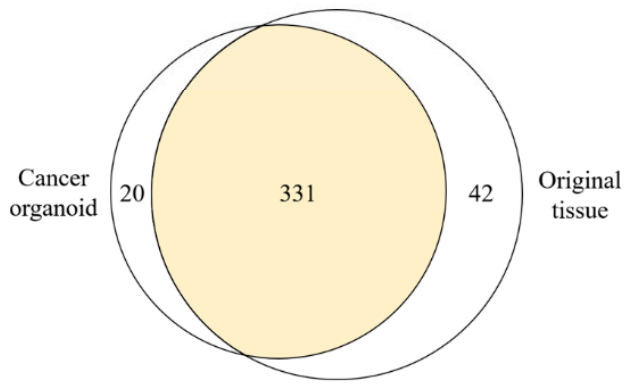

E

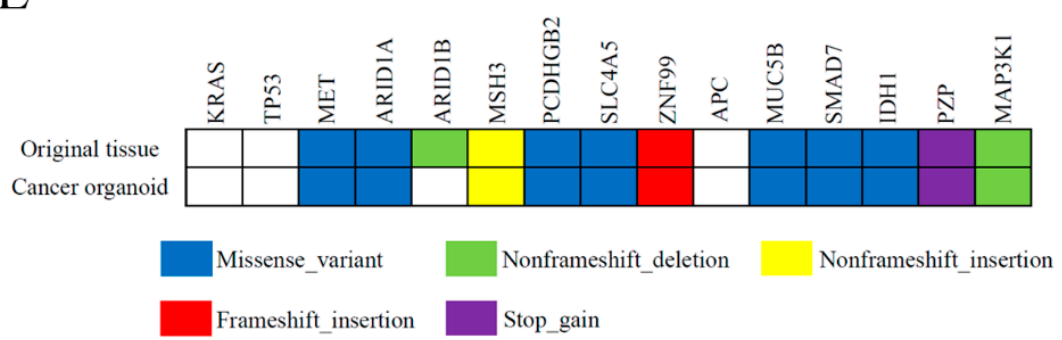

Figure 5. Whole exome sequencing of original tissue and the cancer organoid. (A,B) Circos plots of the original tissue and cancer organoid. (C) The distribution of base substitutions in the organoid and original tissue. (D) Venn diagram demonstrating $89 \%$ overlap of single nucleotide variants between the original tissue and cancer organoid. (E) Representative variants in the original tissue and cancer organoid. 


\subsection{Drug Screening}

The established organoid was used for drug screening and tested with the chemotherapeutic agents, gemcitabine, 5-FU, cisplatin, paclitaxel; and the drugs used for targeted therapies, namely, infigratinib and ivosidenib. Patient 8 received gemcitabine plus albuminbound paclitaxel treatment as conversion therapy for three months. Magnetic resonance images (Figure 6A,B) indicated partial response of the tumor after treatment. These findings were consistent with the results of drug screening using the cancer organoid (Figure 6C). Among the four chemotherapeutic drugs that were tested, gemcitabine and paclitaxel exhibited the strongest inhibitory effects on the cancer organoids, with half-maximal inhibitory concentrations (IC50) of $0.1965 \mu \mathrm{M}$ and $1.697 \mu \mathrm{M}$, respectively (Figure 6D). Moreover, the inhibitor of the isocitrate dehydrogenase 1 (IDH1) family, ivosidenib, exhibited a strong inhibitory effect on the IHCC organoid, with an IC50 of $0.0226 \mu \mathrm{M}$ (Figure 6E,F). This could likely be attributed to the IDH1 missense variant as seen in Figure 5E. On the other hand, the fibroblast growth factor receptor (FGFR) inhibitor, infigratinib, exhibited a marginal inhibitory effect on the cancer organoid.

A

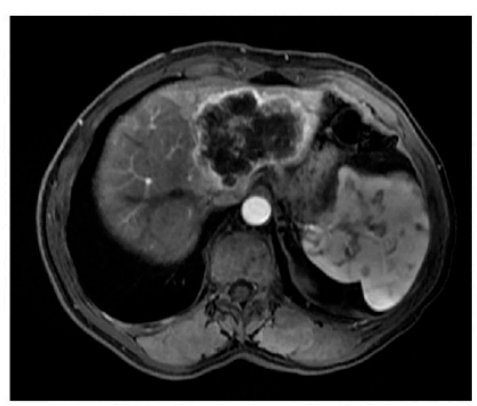

$\mathrm{C}$

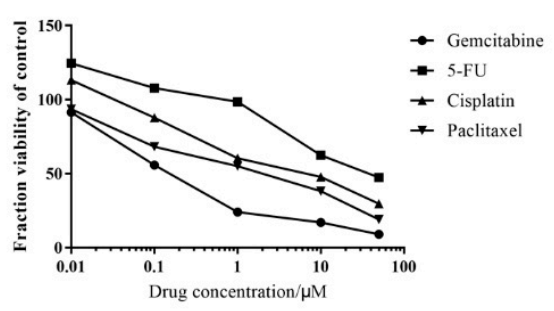

E

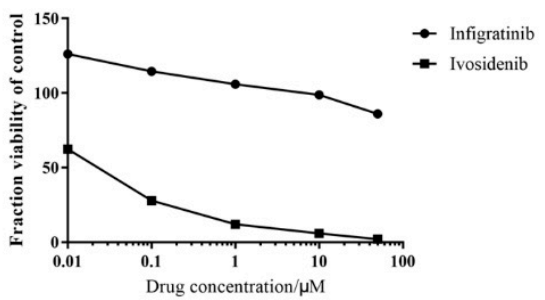

B

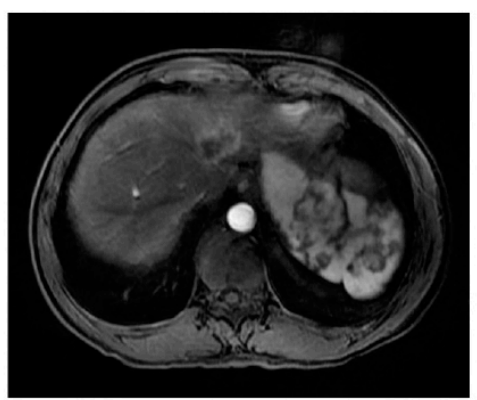

$\mathrm{D}$

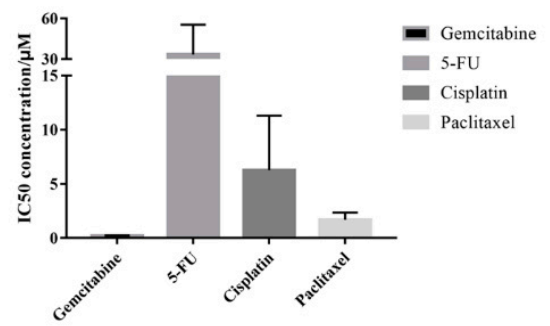

$\mathrm{F}$

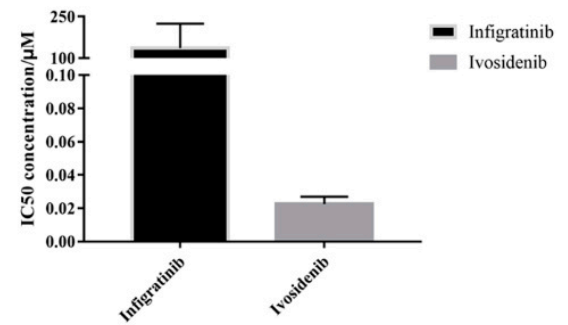

Figure 6. Drug screening of cancer organoid and validation of clinical case. (A,B) Magnetic resonance images of patient 8 at admission (A) and after receiving treatment of gemcitabine plus albuminbound paclitaxel for three months (B). (C-F) Dose-response curves and half-maximal inhibitory concentrations (IC50) of the derived IHCC organoid treated with conventional chemotherapeutic drugs $(\mathbf{C}, \mathbf{D})$ and targeted drugs $(\mathbf{E}, \mathbf{F})$. Screening of each drug was performed in triplicate.

\section{Discussion}

In this study, we evaluated the present situation of IHCC conversion therapy in a single study center. The results confirmed the effectiveness of conversion therapy using IHCC; however, the success rate of downstaging using conversion therapy in patients was 
relatively low $(21.4 \%)$, and thereby, warranted an in vitro cell model to direct the treatment pattern. We established a cancer organoid of IHCC and determined that it could mimic the structural characteristics and retain the gene expression profiles of the original IHCC tissues. Moreover, the results obtained after drug screening using the cancer organoid was consistent with the clinical responses of patients, indicating the ability and effectiveness of the organoid to design a treatment pattern for conversion therapy.

Advanced IHCC was previously considered unsuitable for surgical treatment; however, conversion therapy improved the prognosis of patients to some extent. As reported in a systematic review, 27 of the 132 patients across 10 studies were downstaged successfully using conversion therapy and 23 patients were reported to be alive at the last follow-up that was conducted [25]. The survival rate of downstaged patients in the systematic review was $85.2 \%$ and was similar to that obtained in our study (77.8\%). A study by Edeline J. et al. reports that 9 patients were downstaged successfully using radioembolization plus chemotherapy, of which 8 patients survived after 24 months of follow-up [18]. Moreover, Riby D. et al. compared the overall survival of 137 initially resectable patients and 32 successfully downstaged patients, showing no significant difference between them [26]. However, the response rate of conversion therapy is still low. The overall response rate has been reported as $17.3 \%$ in the systematic review and was found to be $21.4 \%$ in our study [25]. The main reason why downstaging failed in 33 of included 42 patients is that tumors in these 33 patients were resistant to received drugs or local treatment. On the other hand, Le Roy B. et al. reported a much higher response rate of 53\% in locally advanced IHCC patients due to a rather high sensitivity of enrolled IHCC to conversion therapy [27]. The most effective treatment pattern was found to be transarterial radioembolization alone, while chemotherapy with or without radioembolization was the most commonly used therapy in our study. The selection of different conversion strategies was based on the suggestions of guidelines combined with personal experience. An improvement in drug selection for chemotherapy would be the key to increase the response rate of conversion therapy and prolong the overall survival of patients with IHCC.

The concept of using cancer organoids was proposed several decades ago and has been used as a model for drug screening in recent years. Ganesh K. et al. found that the drug sensitivity of the cancer organoid-based platform is positively correlated with clinical response [21]. Some other studies have also established the effectiveness of cancer organoids in drug screening. However, a majority of the studies did not correlate the results from drug screening with clinical response $[24,28,29]$, because surgically resected cancer tissues were used as original specimens of the cancer organoids. It is known that the postoperative recurrence of the tumor and the prognosis of patients does not depend solely on the response of the tumor to chemotherapeutic drugs, but is also attributed to the negative margin, lymph node metastases, and the extent of vascular invasion. Based on these factors, the drug screening of cancer organoids derived from surgically resected tissues often poses a challenge to be correlated with the clinical responses in patients. In this study, we used specimens from successfully downstaged patients with IHCC for organoid establishment within 3 weeks, in whom the drug sensitivity to the tumor was confirmed. As expected, gemcitabine and paclitaxel were found to be the most effective drugs in the cancer organoid model, whereas gemcitabine plus albumin-bound paclitaxel induced a partial response in the patient.

A more suitable approach could likely entail the collection of tumor tissues using needle biopsies for organoid establishment in patients with initially unresectable cancer, followed by comparison with the clinical responses obtained based on drug screening. Nuciforo S. et al. attempted this approach in liver cancers and obtained hepatocellular carcinoma organoids and IHCC organoids at relatively low success rates of $33.3 \%$ and $60 \%$, respectively [30]. In this study, our attempts at establishing an organoid using needle biopsy tissues proved to be unsuccessful. This procedure requires a precise puncture depth at a specific site containing an adequate amount of cancerous tissue. IHCC tissues containing extensive stromal cells may lead to multiple punctures and pose a high risk 
of bleeding. Therefore, the method to successfully obtain specimens to establish cancer organoids needs improvement.

Targeted therapy for IHCC has been explored for several decades. FGFR and IDH1 inhibitors are the two most studied class of compounds in recent times. FGFR variants are often observed in several cancer types including IHCC. FGFR-containing fusions caused by chromosomal translocations have been detected in 10-15\% of the cases of IHCC and are known to play a vital role in the proliferation and metastasis of IHCC [31,32]. It has been reported that treatment with the FGFR inhibitor, infigratinib, may be effective in patients with FGFR-driven HCC [33]. Unfortunately, the IHCC samples in this study were not found to harbor the FGFR variant, and infigratinib did not exert a significant effect on the cancer organoid in vitro. On the contrary, IHD1, another common variant gene in IHCC accounting for up to $25 \%$ of cases [34], was detected in IHCC tissues and the derived organoids in our study. Accordingly, the IDH1 inhibitor, ivosidenib, showed remarkable inhibitory effects on the cancer organoid in vitro. Results of a phase 1 study suggest that ivosidenib is a well-tolerated option in patients with IDH1-variant cholangiocarcinoma [35], and phase 3 study shows progression-free survival was significantly improved with ivosidenib compared with placebo in advanced, IDH1-mutant cholangiocarcinoma [36]. In our study, we found that the organoids could be helpful in providing directions for targeted therapy by retaining some key genetic variants of the original tissue.

Our study had several limitations. First, it comprised only a small sample size of 42 patients receiving conversion therapy and the period of follow up was relatively short, which may lead to bias in data analysis. Moreover, many IHCC patients admitted to our hospital were diagnosed as metastatic stage and refused any treatment due to financial pressure or other reasons, which may result in some referral bias. A multi-center study would have been a better choice considering the low incidence of IHCC. Secondly, the cancer organoid established in the current study was relatively small compared to that in our previous study, which posed several challenges while performing immunohistochemistry and immunofluorescence analyses $[23,24]$. Therefore, we further used WES to compare the gene expression profiles of the organoid and the original tissue. Thirdly, the organoid in this study was established based on a conventional procedure [37]. Such organoids cannot be used for drug screening from the point of immunotherapy owing to a lack of immune cells. Novel methods to establish cancer organoids and reserve stromal cells have been recently proposed, which may provide a feasible approach to investigate the effects of immunotherapy in cancer [38].

\section{Conclusions}

To conclude, conversion therapy, as a novel treatment modality in IHCC, can result in an improvement in overall survival and better prognosis of patients. The low success rate of downstaging in conversion therapy continues to pose a challenge. The therapeutic approach using cancer organoids could likely play a vital role in directing conversion therapy owing to its ability to retain the original genetic expression profile and drug sensitivity.

Author Contributions: Conceptualization, Z.W., Y.J., L.Z. and J.L.; methodology, Z.W. and Y.J.; software, Z.W. and Y.J.; validation, Z.W. and Y.J.; investigation, Z.W., Y.J., Y.G., Z.T., X.Z., D.Y. and Y.Y.; resources, Z.W., Y.J., Y.G., Z.T., X.Z., D.Y. and Y.Y.; data curation, Z.W. and Y.J.; Writing-Original draft preparation, Z.W. and Y.J.; Writing-Review and editing, S.P., L.Z. and J.L.; visualization, L.Z. and J.L.; supervision, L.Z. and J.L.; project administration, J.L.; funding acquisition, J.L. All authors have read and agreed to the published version of the manuscript.

Funding: This research was funded by the National Natural Science Foundation of China (81770614) and the Natural Science Foundation of Zhejiang Province (LQ21H030005).

Institutional Review Board Statement: The study was conducted according to the guidelines of the Declaration of Helsinki, and approved by the ethics committee of the Second Affiliated Hospital, Zhejiang University, School of Medicine (No. 2019-408).

Informed Consent Statement: Informed consent from patients was obtained before tissue collection. 
Data Availability Statement: The data presented in this study are available on request from the corresponding author. The data are not publicly available due to the privacy of enrolled patient.

Conflicts of Interest: The authors declare no conflict of interest.

\section{References}

1. Zhang, X.F.; Xue, F.; Dong, D.H.; Weiss, M.; Popescu, I.; Marques, H.P.; Aldrighetti, L.; Maithel, S.K.; Pulitano, C.; Bauer, T.W.; et al. Number and station of lymph node metastasis after curative-intent resection of intrahepatic cholangiocarcinoma impact prognosis. Ann. Surg. 2020. [CrossRef]

2. $\quad$ Sirica, A.E.; Gores, G.J.; Groopman, J.D.; Selaru, F.M.; Strazzabosco, M.; Wei Wang, X.; Zhu, A.X. Intrahepatic cholangiocarcinoma: Continuing challenges and translational advances. Hepatology 2019, 69, 1803-1815. [CrossRef] [PubMed]

3. Mazzaferro, V.; Gorgen, A.; Roayaie, S.; Droz Dit Busset, M.; Sapisochin, G. Liver resection and transplantation for intrahepatic cholangiocarcinoma. J. Hepatol. 2020, 72, 364-377. [CrossRef]

4. Chen, L.; Zeng, F.; Yao, L.; Fang, T.; Liao, M.; Long, J.; Xiao, L.; Deng, G. Nomogram based on inflammatory indices for differentiating intrahepatic cholangiocarcinoma from hepatocellular carcinoma. Cancer Med. 2020, 9, 1451-1461. [CrossRef] [PubMed]

5. Martin, S.P.; Drake, J.; Wach, M.M.; Ruff, S.M.; Diggs, L.P.; Wan, J.Y.; Good, M.L.; Dominguez, D.A.; Ayabe, R.I.; Glazer, E.S.; et al. Resection and chemotherapy is the optimal treatment approach for patients with clinically node positive intrahepatic cholangiocarcinoma. HPB 2020, 22, 129-135. [CrossRef]

6. Zhou, R.; Lu, D.; Li, W.; Tan, W.; Zhu, S.; Chen, X.; Min, J.; Shang, C.; Chen, Y. Is lymph node dissection necessary for resectable intrahepatic cholangiocarcinoma? A systematic review and meta-analysis. HPB 2019, 21, 784-792. [CrossRef] [PubMed]

7. Bismuth, H.; Adam, R.; Levi, F.; Farabos, C.; Waechter, F.; Castaing, D.; Majno, P.; Engerran, L. Resection of nonresectable liver metastases from colorectal cancer after neoadjuvant chemotherapy. Ann. Surg. 1996, 224, 509-520. [CrossRef] [PubMed]

8. Wang, M.; Xu, Y.; Yang, M.; Jiang, D.; Chen, Y.; Jiang, J.; Chen, Z.; Yang, L.; Huang, D. Conversion therapy for advanced pancreatic cancer: The case series and literature review. Front Pharmacol. 2020, 11, 579239. [CrossRef]

9. Yoshida, K.; Yamaguchi, K.; Okumura, N.; Tanahashi, T.; Kodera, Y. Is conversion therapy possible in stage iv gastric cancer: The proposal of new biological categories of classification. Gastric Cancer 2016, 19, 329-338. [CrossRef] [PubMed]

10. Wei, M.; Shi, S.; Hua, J.; Xu, J.; Yu, X.; Chinese Study Group for Pancreatic Cancer. Simultaneous resection of the primary tumour and liver metastases after conversion chemotherapy versus standard therapy in pancreatic cancer with liver oligometastasis: Protocol of a multicentre, prospective, randomised phase iii control trial (cspac-1). BMJ Open 2019, 9, e033452. [CrossRef] [PubMed]

11. Ghiringhelli, F.; Lorgis, V.; Vincent, J.; Ladoire, S.; Guiu, B. Hepatic arterial infusion of gemcitabine plus oxaliplatin as secondline treatment for locally advanced intrahepatic cholangiocarcinoma: Preliminary experience. Chemotherapy 2013, 59, 354-360. [CrossRef] [PubMed]

12. Kato, A.; Shimizu, H.; Ohtsuka, M.; Yoshidome, H.; Yoshitomi, H.; Furukawa, K.; Takeuchi, D.; Takayashiki, T.; Kimura, F.; Miyazaki, M. Surgical resection after downsizing chemotherapy for initially unresectable locally advanced biliary tract cancer: A retrospective single-center study. Ann. Surg. Oncol. 2013, 20, 318-324. [CrossRef]

13. Mouli, S.; Memon, K.; Baker, T.; Benson, A.B., 3rd; Mulcahy, M.F.; Gupta, R.; Ryu, R.K.; Salem, R.; Lewandowski, R.J. Yttrium-90 radioembolization for intrahepatic cholangiocarcinoma: Safety, response, and survival analysis. J. Vasc. Interv. Radiol. 2013, 24, 1227-1234. [CrossRef]

14. Rayar, M.; Sulpice, L.; Edeline, J.; Garin, E.; Levi Sandri, G.B.; Meunier, B.; Boucher, E.; Boudjema, K. Intra-arterial yttrium-90 radioembolization combined with systemic chemotherapy is a promising method for downstaging unresectable huge intrahepatic cholangiocarcinoma to surgical treatment. Ann. Surg. Oncol. 2015, 22, 3102-3108. [CrossRef] [PubMed]

15. Servajean, C.; Gilabert, M.; Piana, G.; Monges, G.; Delpero, J.R.; Brenot, I.; Raoul, J.L. One case of intrahepatic cholangiocarcinoma amenable to resection after radioembolization. World J. Gastroenterol. 2014, 20, 5131-5134. [CrossRef] [PubMed]

16. Tran, T.B.; Bal, C.K.; Schaberg, K.; Longacre, T.A.; Chatrath, B.S.; Poultsides, G.A. Locally advanced intrahepatic cholangiocarcinoma: Complete pathologic response to neoadjuvant chemotherapy followed by left hepatic trisectionectomy and caudate lobectomy. Dig. Dis. Sci. 2015, 60, 3226-3229. [CrossRef] [PubMed]

17. Uji, M.; Mizuno, T.; Ebata, T.; Sugawara, G.; Igami, T.; Uehara, K.; Nagino, M. A case of advanced intrahepatic cholangiocarcinoma accidentally, but successfully, treated with capecitabine plus oxaliplatin (capox) therapy combined with bevacizumab: A case report. Surg. Case Rep. 2016, 2, 63. [CrossRef] [PubMed]

18. Edeline, J.; Touchefeu, Y.; Guiu, B.; Farge, O.; Tougeron, D.; Baumgaertner, I.; Ayav, A.; Campillo-Gimenez, B.; Beuzit, L.; Pracht, M.; et al. Radioembolization plus chemotherapy for first-line treatment of locally advanced intrahepatic cholangiocarcinoma: A phase 2 clinical trial. JAMA Oncol. 2019, 6, 51-59. [CrossRef] [PubMed]

19. Roodsant, T.; Navis, M.; Aknouch, I.; Renes, I.B.; van Elburg, R.M.; Pajkrt, D.; Wolthers, K.C.; Schultsz, C.; van der Ark, K.C.H.; Sridhar, A.; et al. A human $2 \mathrm{~d}$ primary organoid-derived epithelial monolayer model to study host-pathogen interaction in the small intestine. Front. Cell. Infect. Microbiol. 2020, 10, 272. [CrossRef] [PubMed]

20. Tuveson, D.; Clevers, H. Cancer modeling meets human organoid technology. Science 2019, 364, 952-955. [CrossRef] [PubMed]

21. Ganesh, K.; Wu, C.; O’Rourke, K.P.; Szeglin, B.C.; Zheng, Y.; Sauve, C.G.; Adileh, M.; Wasserman, I.; Marco, M.R.; Kim, A.S.; et al A rectal cancer organoid platform to study individual responses to chemoradiation. Nat. Med. 2019, 25, 1607-1614. [CrossRef] 
22. Frappart, P.O.; Walter, K.; Gout, J.; Beutel, A.K.; Morawe, M.; Arnold, F.; Breunig, M.; Barth, T.F.; Marienfeld, R.; Schulte, L.; et al. Pancreatic cancer-derived organoids-a disease modeling tool to predict drug response. United Eur. Gastroenterol. J. 2020, 8, 594-606. [CrossRef] [PubMed]

23. Broutier, L.; Mastrogiovanni, G.; Verstegen, M.M.; Francies, H.E.; Gavarro, L.M.; Bradshaw, C.R.; Allen, G.E.; Arnes-Benito, R.; Sidorova, O.; Gaspersz, M.P.; et al. Human primary liver cancer-derived organoid cultures for disease modeling and drug screening. Nat. Med. 2017, 23, 1424-1435. [CrossRef] [PubMed]

24. Driehuis, E.; van Hoeck, A.; Moore, K.; Kolders, S.; Francies, H.E.; Gulersonmez, M.C.; Stigter, E.C.A.; Burgering, B.; Geurts, V.; Gracanin, A.; et al. Pancreatic cancer organoids recapitulate disease and allow personalized drug screening. Proc. Natl. Acad. Sci. USA 2019, 116, 26580-26590. [CrossRef] [PubMed]

25. Fruscione, M.; Pickens, R.C.; Baker, E.H.; Martinie, J.B.; Iannitti, D.A.; Hwang, J.J.; Vrochides, D. Conversion therapy for intrahepatic cholangiocarcinoma and tumor downsizing to increase resection rates: A systematic review. Curr. Probl. Cancer 2020, 45, 100614. [CrossRef] [PubMed]

26. Riby, D.; Mazzotta, A.D.; Bergeat, D.; Verdure, L.; Sulpice, L.; Bourien, H.; Lievre, A.; Rolland, Y.; Garin, E.; Boudjema, K.; et al. Downstaging with radioembolization or chemotherapy for initially unresectable intrahepatic cholangiocarcinoma. Ann. Surg. Oncol. 2020, 27, 3729-3737. [CrossRef] [PubMed]

27. Le Roy, B.; Gelli, M.; Pittau, G.; Allard, M.A.; Pereira, B.; Serji, B.; Vibert, E.; Castaing, D.; Adam, R.; Cherqui, D.; et al. Neoadjuvant chemotherapy for initially unresectable intrahepatic cholangiocarcinoma. Br. J. Surg. 2018, 105, 839-847. [CrossRef]

28. Bruun, J.; Kryeziu, K.; Eide, P.W.; Moosavi, S.H.; Eilertsen, I.A.; Langerud, J.; Rosok, B.I.; Totland, M.Z.; Brunsell, T.H.; Pellinen, T.; et al. Patient-derived organoids from multiple colorectal cancer liver metastases reveal moderate intra-patient pharmacotranscriptomic heterogeneity. Clin. Cancer Res. 2020, 26, 4107-4119. [CrossRef]

29. Li, X.; Pan, B.; Song, X.; Li, N.; Zhao, D.; Li, M.; Zhao, Z. Breast cancer organoids from a patient with giant papillary carcinoma as a high-fidelity model. Cancer Cell Int. 2020, 20, 86. [CrossRef]

30. Nuciforo, S.; Fofana, I.; Matter, M.S.; Blumer, T.; Calabrese, D.; Boldanova, T.; Piscuoglio, S.; Wieland, S.; Ringnalda, F.; Schwank, G.; et al. Organoid models of human liver cancers derived from tumor needle biopsies. Cell Rep. 2018, 24, $1363-1376$. [CrossRef]

31. Lamberti, D.; Cristinziano, G.; Porru, M.; Leonetti, C.; Egan, J.B.; Shi, C.X.; Buglioni, S.; Amoreo, C.A.; Castellani, L.; Borad, M.J.; et al. Hsp90 inhibition drives degradation of fgfr2 fusion proteins: Implications for treatment of cholangiocarcinoma. Hepatology 2019, 69, 131-142. [CrossRef] [PubMed]

32. Zhou, Y.; Wu, C.; Lu, G.; Hu, Z.; Chen, Q.; Du, X. Fgf/fgfr signaling pathway involved resistance in various cancer types. J. Cancer 2020, 11, 2000-2007. [CrossRef] [PubMed]

33. Huynh, H.; Lee, L.Y.; Goh, K.Y.; Ong, R.; Hao, H.X.; Huang, A.; Wang, Y.; Graus Porta, D.; Chow, P.; Chung, A. Infigratinib mediates vascular normalization, impairs metastasis, and improves chemotherapy in hepatocellular carcinoma. Hepatology 2019, 69, 943-958. [CrossRef] [PubMed]

34. Boscoe, A.N.; Rolland, C.; Kelley, R.K. Frequency and prognostic significance of isocitrate dehydrogenase 1 mutations in cholangiocarcinoma: A systematic literature review. J. Gastrointest. Oncol. 2019, 10, 751-765. [CrossRef] [PubMed]

35. Lowery, M.A.; Burris, H.A., 3rd; Janku, F.; Shroff, R.T.; Cleary, J.M.; Azad, N.S.; Goyal, L.; Maher, E.A.; Gore, L.; Hollebecque, A.; et al. Safety and activity of ivosidenib in patients with idh1-mutant advanced cholangiocarcinoma: A phase 1 study. Lancet Gastroenterol. Hepatol. 2019, 4, 711-720. [CrossRef]

36. Abou-Alfa, G.K.; Macarulla, T.; Javle, M.M.; Kelley, R.K.; Lubner, S.J.; Adeva, J.; Cleary, J.M.; Catenacci, D.V.; Borad, M.J.; Bridgewater, J.; et al. Ivosidenib in idh1-mutant, chemotherapy-refractory cholangiocarcinoma (claridhy): A multicentre, randomised, double-blind, placebo-controlled, phase 3 study. Lancet Oncol. 2020, 21, 796-807. [CrossRef]

37. Driehuis, E.; Kretzschmar, K.; Clevers, H. Establishment of patient-derived cancer organoids for drug-screening applications. Nat. Protoc. 2020, 15, 3380-3409. [CrossRef] [PubMed]

38. Neal, J.T.; Li, X.; Zhu, J.; Giangarra, V.; Grzeskowiak, C.L.; Ju, J.; Liu, I.H.; Chiou, S.H.; Salahudeen, A.A.; Smith, A.R.; et al. Organoid modeling of the tumor immune microenvironment. Cell 2018, 175, 1972-1988 e1916. [CrossRef] 